\title{
Value of Black-blood MR Angiography Generated from Vessel Wall Imaging in the Assessment of Stented Intracranial Arteries
}

\author{
Kazuhiro Tsuchiya ${ }^{1)}$, Miho Gomyo 1), Masaaki Shojima ${ }^{2)}$, Kenichi Yokoyama ${ }^{3)}$
}

1) Department of Radiology, Saitama Medical Center, Saitama Medical University, Kawagoe, Japan

2) Department of Neurosurgery, Saitama Medical Center, Saitama Medical University, Kawagoe, Japan

3) Department of Radiology, Kyorin University, Tokyo, Japan

\section{Background}

Intracranial stents are often used to assist embolization of widenecked aneurysms $(1,2)$. Although the long-term benefit has been denied, stenting may still be beneficial in some patients with intracranial artery stenosis in combination with percutaneous transluminal angioplasty $(3,4)$. Stents cause endothelial hyperplasia or thrombosis. For the assessment of stented intracranial arteries, DSA, 3D-TOF MRA and CTA have been employed. However, their clinical value is limited due to invasiveness and/or artifacts including susceptibility artifacts, radiofrequency shielding and turbulent/laminar flow $(6,7)$.

\section{Purpose}

For intracranial vessel wall imaging (VWI), we have used a SPACE (sampling perfection with application optimized contrast using different flip angle evolution; Siemens Healthineers) sequence based on a fast spin-echo technique with variable flip angle refocusing pulses. Therefore, we hypothesized that VWI may be useful for the accurate evaluation of the stented artery. In this study, we comparatively evaluated the depiction of stented artery between conventional 3D-TOF MRA and black-blood MRA (BB-MRA) generated from VWI data obtained using the SPACE sequence in order to prove the hypothesis.

\section{Materials and Methods}

Imaging

$\checkmark$ 3T MR scanner (Verio, Siemens Healthineers)

\begin{tabular}{lll}
\hline Sequence & 3D-TOF MRA & UWI and BB-MRA \\
Receiver coil & 3D FFE & SPACE \\
Repetition time/Echo time & $23 / 3.95 \mathrm{msec}$ & $32 \mathrm{ch}$ head coil \\
Matrix & $318 \times 448$ & $270 / 11 \mathrm{msec}$ \\
Field of view & 200 & 240 \\
Slice thickness/number & $0.6 \mathrm{~mm} / 120$ & $0.9 \mathrm{~mm} / 192$ \\
Scan time & $6 \mathrm{~min} 30 \mathrm{sec}$ & $7 \mathrm{~min} 35 \mathrm{sec}$ \\
Image processing & MIP & $\begin{array}{l}\text { minlP } \\
\text { slice thickness, } 30 \mathrm{~mm} \\
\text { slice interval, } 2 \mathrm{~mm}\end{array}$ \\
\hline
\end{tabular}

Image analysis

(1) Contrast ratio

$\checkmark$ Signal intensity (SI) of the central part of stented artery, the normal artery proximal to the stent and the normal white matter were measured on source images.

$\checkmark$ The contrast ratio $(\mathrm{CR})=\left(\mathrm{SI}_{\text {white matter }}-\mathrm{SI}_{\text {stented artery }}\right) /(\mathrm{SI}$ white matter $\left.+S I_{\text {proximal artery }}\right)$

(2) Vessel diameter

$\checkmark$ For both stented artery and normal artery proximal to the stent, the profiling curve was obtained in cross section for each artery on source images using the ImageJ software by manual plotting. The full width at half maximum (FWHM) was regarded as the diameter.

$\checkmark$ The relative diameter index $(\mathrm{DI})=$ the diameter (stented artery) / (normal artery proximal to the stent)

(3) Visual assessment

$\checkmark$ We visually assessed depiction of the artery at the poststenting site using the following four-grade visual assessment score.

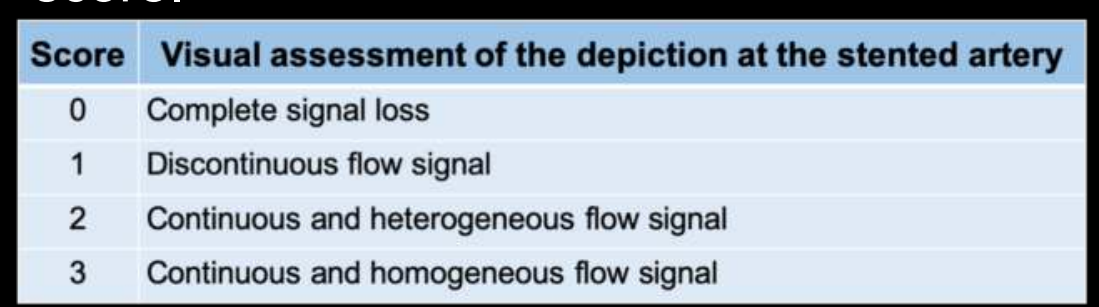

Results

\section{(1) Contrast ratio}

On 3D-TOF MRA, the CR of the stented artery was significantly lower than that of the normal artery $(0.43$ vs. $0.67, P<0.05)$. On BB-MRA, there was no significant difference in the CR for both stented artery and normal artery ( 0.90 vs. $0.90, P=0.926)$.

\section{Results (cont'd)}

(2) Vessel diameter

Significant difference was present in the relative diameter indexes (Dls) between 3D-TOF and BB-MRA (0.58 vs. 0.96, P<0.01). On 3D-TOF MRA, the diameter of the stented artery was smaller than that of the normal artery in all cases (the average ratio $=0.56$ ). However, there was no difference in the diameter on BB-MRA (the average ratio $=0.94$ ).

(3) Visual assessment

There was significant difference between 3D-TOF MRA and BB-MRA (1.0 vs. $3.0, \mathrm{P}<0.05)$.

On 3D-TOF MRA, the flow signal tended to be discontinuous or heterogeneous. On the BB-MRA, the flow signal was homogeneous and continuous in all cases.

Representative Case (68-y.0. female after stent-assisted coil embolization for a left MCA aneurysm)

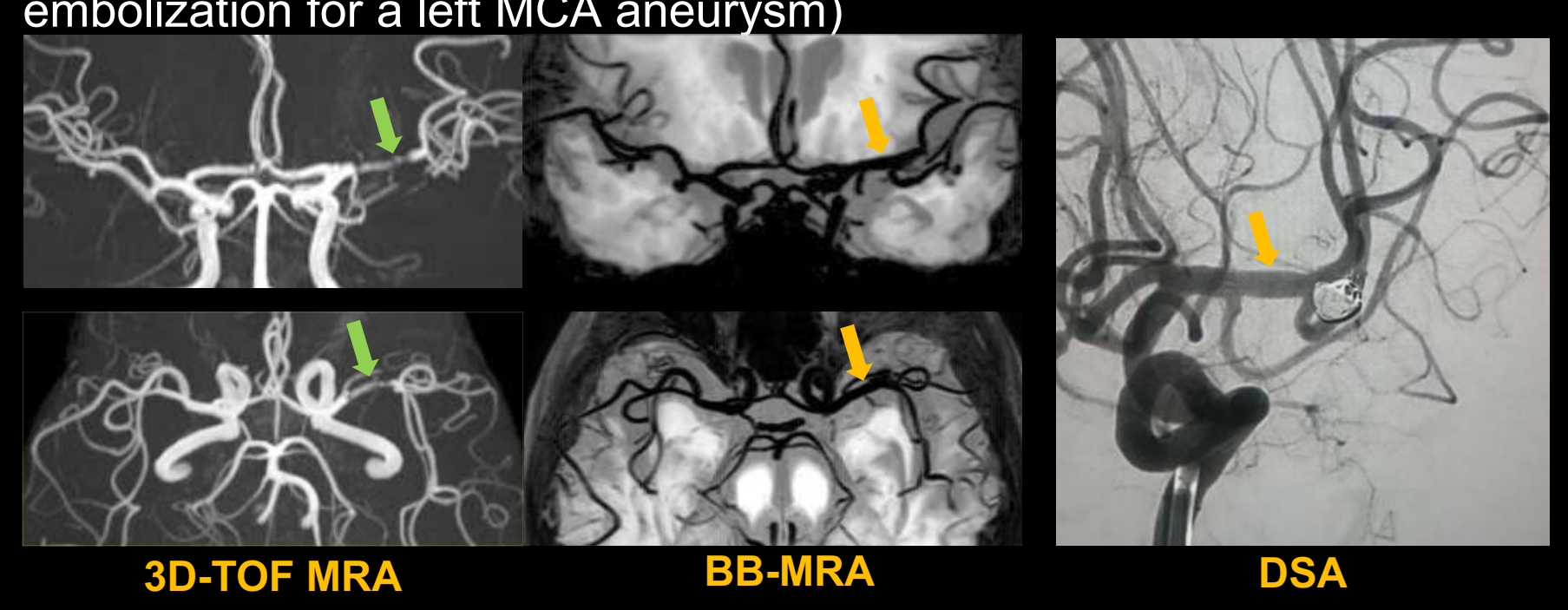

On 3D-TOF MRA, left M1 seems to show irregularity and stenosis. On BB-MRA, however, the M1 is apparently patent. DSA shows patent M1 lumen that is well corresponding with BB-MRA.

\section{Discussion}

The CR of the stented artery was significantly higher than that of the normal artery on BB-MRA than on 3D-TOF MRA. Similarly, visual assessment showed that BB-MRA is better than 3D-TOF MRA in signal homogeneity and continuity. These are probably due to signal loss of the stented artery caused by susceptibility artifacts in 3D-TOF MRA using a gradient-echo sequence, while BB-MRA is free from such artifacts as it uses a spin-echo sequence.

On 3D-TOF MRA, shielding effects, which induce currents in the stent wall, reportedly more affects signal of the stented artery than susceptibility artifacts (5). In a study that evaluated difference in signal loss among several kinds of stents, LVIS stent has been reported to show the minimal degree (6). Our group previously performed a similar study in clinical cases and it showed that, although difference was statistically insignificant, the CR of LVIC stent tended to be low compared with Neuroform atlas (7). This suggests that geometry and orientation of stents affect susceptibility artifacts leading to more signal loss than that caused by shielding effects.

Anyway, judging from our results of the assessment of relative DI, BBMRA seems to be more accurate than 3D-TOF MRA in assessing lumen of the stented arteries. Although not evaluated in the present study, source images of WWI before performing minIP could also be informative.

\section{Conclusion}

BB-MRA generated from VWI could be useful for the follow-up study of stent-assisted treatment of intracranial arterial diseases.

\footnotetext{
References

1. Henkes H, Bose A. Felber S, et al. Interv Neuroradiol 30;107-119, 2002

2. Higashida RT. Halbach VV, Dowd CF, et al. AJNR Am J Neuroradiol 26;1751-1756, 2005

3. Jia BX, Feng L, Liebeskind DS, et al. J Neurolntervent Surg 10;746-750, 2018

4. Alexander MJ, Zauner A, Chaloupka JC, et al. Stroke 50;889-894, 2019

5. Wang Y, Truong TN, Yen C, et al. Magn Reson Med 49;972-976, 2003

6. Hayashi T, Fukuda A. Nihon Hoshasen Gijutsu Gakkai Zasshi 75;747-753, 2019

7. Gomyo M, Tsuchiya K, Hosoi S, et al. Presented at the 47th Annual Meeting of Japanese Society for Magnetic Resonance in Medicine (JSMRM 2019).
} 